\title{
Laboreal
}

Volume $13 \mathrm{~N}^{\circ} 2$ | 2017

Varia

\section{J.-M. Lahy crítica de Taylor, ¿un recurso para pensar el trabajo de hoy en día?}

J.-M. Lahy crítico de Taylor, um recurso para pensar o trabalho hoje?

J.-M. Lahy critique de Taylor, une ressource pour penser le travail aujourd'hui ?

J.-M. Lahy criticises taylor, a resource to think today's work?

Jean-Luc Tomás y Maria laneva

Traductor. Cecilia De la Garza, Patricio Nusshold y Mario Poy

(2) OpenEdition

Journals

Edición electrónica

URL: http://journals.openedition.org/laboreal/348

DOI: 10.4000/laboreal.348

ISSN: $1646-5237$

Editor

Universidade do Porto

Referencia electrónica

Jean-Luc Tomás y Maria laneva, « J.-M. Lahy crítica de Taylor, ¿un recurso para pensar el trabajo de hoy en día? », Laboreal [En línea], Volume 13 N² | 2017, Publicado el 01 diciembre 2017, consultado el 23 septiembre 2020. URL : http://journals.openedition.org/laboreal/348 ; DOI : https://doi.org/ 10.4000/laboreal.348

Este documento fue generado automáticamente el 23 septiembre 2020

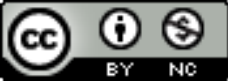

Laboreal está licenciado com uma Licença Creative Commons - Atribuição-NãoComercial 4.0 Internacional. 


\title{
J.-M. Lahy crítica de Taylor, ¿un recurso para pensar el trabajo de hoy en día?
}

\author{
J.-M. Lahy crítico de Taylor, um recurso para pensar o trabalho hoje? \\ J.-M. Lahy critique de Taylor, une ressource pour penser le travail aujourd'hui? \\ J.-M. Lahy criticises taylor, a resource to think today's work?
}

Jean-Luc Tomás y Maria laneva

Tradución : Cecilia De la Garza, Patricio Nusshold y Mario Poy

\section{REFERENCIA}

Lahy, J.-M. (1916). le système Taylor el la physiologie du travail professionnel (introduction). Paris: Masson.

\section{NOTA DEL EDITOR}

http://dx.doi.org/10.15667/laborealxiii0217jles

Manuscrito recibido en: Septiembre/2017

Aceptado tras peritaje: Noviembre/2017

$1 \quad$ El libro de J.-M. Lahy (1916) - Le système Taylor et la physiologie du travail professionnel (El sistema Taylor y la fisiología del trabajo profesional) - tiene más de un siglo, pero sigue siendo uno de los más pertinentes sobre el taylorismo. Su contenido es conocido y ha sido comentado a menudo (véase por ejemplo, Friedmann, 1946). Nuestra contribución se enfocara sobre todo en la introducción de esta obra y tratará de explicar como, el propósito de Lahy, sigue siendo de actualidad. Recordamos en primer lugar ciertos elementos bibliográficos del autor así como el contexto social, económico y político a principios del siglo 20. Este marca en efecto un cambio en la relación entre las ciencias 
del trabajo y la organización de la producción, que algunos autores (ver por ejemplo, Vatin, 1999) califican de "giro socio-epistemológico". Los debates alrededor de la doctrina taylorista acompañan una reflexión acerca del surgimiento y los modos de existencia de una ciencia al servicio del desarrollo industrial, iniciados ya de manera sustancial en Francia y en Europa (Vatin, 2008). En segundo lugar, nos apoyaremos en el texto para abrir una discusión sobre las relaciones, siempre complejas, entre la psicología y la empresa. Al centro de dichas relaciones, encontramos hoy como ayer, la promesa de un compromiso social y organizacional renovado.

\section{Elementos biográficos}

2 El itinerario científico de Lahy (1872-1943) es en varios aspectos original. Para empezar, su biografía permanece incompleta en parte y comienza en general en 1901 cuando tiene ya 29 años. En un artículo reciente Turbiaux (2006) menciona sin embargo que Lahy ingresa a los 20 años a la oficina de Correos y Telégrafos, e integra las oficinas ambulantes del Sur Oeste de Lyon después de su servicio militar. En cuanto a su formación intelectual, queda aún hoy en día por establecer con exactitud. Al parecer siguió los cursos de Théodule Ribot y de su sucesor, Pierre Janet, en el Colegio de Francia antes de 1901. Según Henri Piéron, Lahy habría asistido a clases de fisiología de François-Franck, en esa misma institución. Además, y durante el mismo periodo, se forma en sociología con Durkheim por medio de una lectura detenida del autor. De origen humilde, sin diplomas universitarios, Lahy se casa en 1901 con Marie-Blanche Trouillet a quien le debe en parte su ascenso social. En efecto, Lahy se inicia a la francmasonería con un tío de su esposa, lo cual le permitirá volverse un miembro sumamente activo de la logia Gran Oriente de Francia.

3 La red de francmasonería le facilita sin duda el encuentro con Edouard Toulouse, en aquel entonces jefe de medicina en el asilo de Villejuif y al origen de la institucionalización del laboratorio de Psicología experimental vinculado a la Escuela Practica de Altos Estudios (EPHE ${ }^{1}$ ). En 1901, Lahy se vuelve uno de los alumnos de ese laboratorio -uno de los más eminentes en psicología científica- y se forma con Nicolas Vaschide y Henri Piéron, en los métodos experimentales. De hecho, es con Vaschide, con quien firma su primer artículo científico en 1902.

4 Al mismo tiempo, Lahy sigue un curso en sociología con Marcel Mauss en la EPHE. Después de defender su tesis sobre las sociedades secretas en Melanesia, obtiene un solo título universitario ${ }^{2}$ en 1907.

5 Sin remuneración por sus trabajos de investigación, Lahy, gracias a la red de francmasonería -y en particular por medio del hermano du esposa-, participa en las obras de varios ministerios y obtiene un puesto de recaudador de impuestos en 1908, en el que se quedará hasta du jubilación en 1928. Sumamente comprometido en el Gran Oriente, solicitado profesionalmente por sus diferentes empleos, Lahy llevó su trabajo de investigación de manera paralela.

6 Finalmente, y mencionando las ultimas originalidades -sin pretensión de exhaustividad-, la pertenencia del "autodidacta" Lahy a dos disciplinas científicas (o tres: la sociología, la psicología y la fisiología) le abrió la posibilidad de adaptar los métodos psicofisiológicos a las cuestiones sociológicas de las profesiones. Después de Toulouse y de su célebre estudio sobre Émile Zola, Lahy va a dedicarse a detectar los 
signos de superioridad profesional, primero con las dactilógrafas (1905), y después con los choferes de tranvías (por ejemplo en 1913). Pero es el siguiente estudio encargado por el Ministerio del trabajo, sobre los obreros tipógrafos $(1909,1910)$, con el que inaugurará una de las primeras intervenciones en psicología en el mundo laboral (Turbiaux, 1999). En efecto, Lahy realiza observaciones de campo, aplica una serie de pruebas fisiológicas y psicológicas, e insiste en sus resultados en las relaciones entre las nuevas organizaciones del trabajo y la fatiga de los obreros. Enfatiza el hecho que la fatiga no solo es de orden fisiológico, sino que también está arraigada en la monotonía de las tareas, en el mantener la atención. Lo que hoy en día parece evidente es, en ese momento crucial, el comienzo de un verdadero nuevo impulso epistemológico, el del trabajo "físico" al trabajo "mental", del obrero como "ser automático" al "ser humano en el trabajo".

\section{Elementos de contexto social, económico y político de principios del siglo 20}

7 La actividad industrial en Francia, entre 1890 y hasta principios de la primera guerra mundial, está marcada por varias crisis y periodos de auge. El peso numérico de los obreros y empleados pasó de tres millones alrededor de 1870 a más de cuatro millones y medio antes de 1914. En el umbral de la primera guerra mundial, la industria empleaba $35 \%$ de la población activa prácticamente tanto como en la agricultura. Ese cambio de escala se acompañó de huelgas y múltiples formas de prácticas contestatarias. De manera paralela, surgen nuevas formas de restricciones disciplinarias. Los reglamentos en los talleres fijan las normas de rendimiento y las de la vida industrial. Para regular el proceso de producción de las fábricas, se crean los comités de dirección. La contratación en aumento del personal de supervisión permite la operacionalización de la división funcional del trabajo. Las maquinas simplifican el trabajo e imponen nuevas reglas de mecanizado, mientras que, por ejemplo, tres cuartos de los obreros de la industria automotriz son verdaderos profesionales de la metalurgia (Cohen, 2001). El cronometraje del trabajo de esos obreros en la industria Renault conlleva a una huelga en febrero y marzo del 1913. Esto resultará un fracaso, los obreros retoman rápidamente el trabajo. Pero el uso mediático de esa huelga va a hacer posible la difusión del modelo de la organización científica del trabajo de Taylor (Fridenson, 1987), el cual conocerá en Francia un auge durante la primera guerra mundial, dados los requerimientos de producción de municiones, ejecutadas por mujeres sin oficio (Hatzfeld, 2011). Esta nueva fábrica, que transforma tanto al trabajo como a las reglas disciplinarias (Perrot, 1983), se realiza en un contexto de alianza entre el poder político y la ciencia. El culto al progreso técnico y científico es así legitimado por el positivismo, que desempeña el rol de garante ideológico y moral. Pero los académicos se encuentran en competencia con los ingenieros, respecto de la aplicación de los métodos de producción y de desempeño en las fábricas, (Gouarné 2013, Paîcheler 1992, Vatin 2008). Es más, los ingenieros intentan, con sus métodos, involucrarse en la resolución de los conflictos sociales.

8 La crítica que le realiza Lahy al taylorismo en $1916^{3}$ es, por lo tanto, parte de este universo compuesto por los movimientos que sacuden y atraviesan a la sociedad francesa. Pero, al elegir como proyecto la pacificación de las relaciones sociales 
interponiendo a la ciencia en la posición de árbitro, deja como herencia a la psicología del trabajo los problemas, las preguntas y las dificultades que deseamos discutir.

\section{Una introducción que sienta hitos y preguntas}

Lahy (1916) organiza el texto introductorio de su libro en tres partes en las que, i) especifica la ruptura epistemológica que ya hemos mencionado, ii) critica de manera explícita el sistema taylorista considerando tres puntos de vista (psicológico, sociológico e industrial), y finalmente, (iii) justifica el rol social de la psicofisiología con el propósito de organizar "verdadera y científicamente" (p. X) el trabajo humano.

\subsection{El trabajador. un ser humano, y no solo un equivalente mecánico}

En la primera parte de su introducción, Lahy sitúa su punto de vista tanto a un nivel epistemológico, como a un nivel social. El "trabajo psico-fisiológico" se resiste a la ley del mayor rendimiento sostenida por la doctrina de Taylor, inclinándose por un proyecto "humanista" de las fuerzas sociales existentes (Le Bianic, 2004). La analogía entre el hombre y la máquina, que el autor aborda y discute desde las primeras líneas de su texto, puede hoy en día sorprender. Para el lector de la época, constituye una "referencia" asociada con una cierta concepción del trabajo "físico" que la psicofisiología viene a renovar (Vatin, 1999). De hecho, si la máquina está programada para actuar, "el ser humano en el trabajo" es, por el contrario, capaz de crear vínculos originales entre sus pensamientos y sus acciones. Por lo tanto, el trabajador no es "un ser automático" (p. VI), el equivalente de una máquina. Las medidas de energía desatienden y descuidan las características psicológicas del trabajo humano. Más aún, ignoran las particulares combinaciones entre pensamiento y actos, excluyendo del análisis la "parte intelectual" (p. IX) del trabajo.

Desde entonces, reducir al trabajador a gestos normalizados y uniformes es, al mismo tiempo, "una pérdida y una ganancia" (página 7). Una pérdida porque los trabajadores se ven privados de su iniciativa, reduciéndolos como personas, y una ganancia porque los gestos se pueden realizar en las mejores condiciones de calidad y rendimiento. Por lo tanto, la mejora de la calidad y el rendimiento siguen siendo un objetivo legítimo para el autor pero bajo ciertas condiciones, a saber, no encerrarse en un proyecto de "perfeccionamiento de la técnica" (p. VII) considerando al obrero un simple "elemento de producción" (Ibidem.). El riesgo de un tal reduccionismo conllevaría afectar el "desarrollo psicológico y fisiológico del hombre normal" (Ibidem.). En otras palabras, los "reformistas" que organizan el trabajo tienen una responsabilidad social, que se mide en la escala del "hombre y la raza" (Ibidem.).

12 Frente a esta constatación de fuertes consecuencias, Lahy hace hincapié en un punto que nos parece importante, dado el contexto actual de la disciplina y de las demandas sociales dirigidas a los psicólogos en ejercicio: "Hay que, por lo tanto, buscar un punto de equilibrio entre el excesivo automatismo humano vinculado al trabajo moderno y la dispersión del pensamiento debido a la ausencia de restricciones" (p.VII). Sobre este punto, Lahy deja abiertas al menos tres preguntas esenciales: (i) ¿Quién busca el "punto de equilibrio"? ii) ¿Cómo buscar este "punto de equilibrio"? Y, finalmente, iii) ¿Cómo 
definir este equilibrio entre "automatismo" y "dispersión del pensamiento", entre las exigencias organizativas y los recursos de la iniciativa?

\subsection{Los errores del taylorismo}

13 La segunda parte de su introducción refiere explícitamente a la nueva organización taylorista, en donde el autor entiende discutir en tres registros: psicológico, sociológico y práctico. El rendimiento buscado por Taylor olvida el "bienestar del trabajador"“(p. VIII). Pero además, este sistema, al asimilar al trabajador a una máquina, de hecho lo somete a un trabajo que aumenta su inferioridad (p. IX) y coloca el debate a nivel de la condición humana. Para contrarrestar este sistema perjudicial, Lahy esboza una propuesta, respondiendo en parte a las preguntas antes mencionadas: la "selección psicológica previa" (p. IX).

14 Para remarcar el error del taylorismo desde un punto de vista sociológico, Lahy introduce un contraste entre el trabajador, atrapado en una organización de trabajo esclavizante y el obreromiembro activo de la sociedad. La llamada organización "científica" del trabajo ignora las posibilidades de invención e iniciativa que posee el ser humano. Por lo tanto, lleva más a una forma de sub-explotación del potencial humano, que a la mejora de la calidad y del rendimiento.

Finalmente, a este desconocimie nto, al límite del desprecio, se le agrega el de un contexto de producción donde el uso creciente de máquinas impone cualidades de "vigilancia, de atención, de adaptación rápida y confiable" (p. IX). El trabajo humano en la fábrica moderna no debe limitarse a considerar al sujeto desde el mero aspecto muscular, ignorando los fenómenos del aprendizaje (Huteau, 2002). Las funciones cognitivas también se ponen a prueba en el trabajo industrial. Es por esto que la máquina humana no solo simplifica el trabajo humano.

\subsection{El lugar del hombre en la organización científica del trabajo}

En la última parte de su introducción, Lahy se vuelve mucho más afirmativo e introduce la figura del psicofisiólogo. Este último tiene el deber, considerando tanto el interés del patrón como del trabajador, de "señalar el lugar que ocupa el ser humano en toda la organización científica del trabajo" (p. X). Son los resultados científicos los que deben proporcionar las claves para comprender los elementos esenciales de la vida obrera y esbozar las líneas de una organización verdaderamente científica del trabajo humano" (p. X. somos nosotros que lo subrayamos). Al posicionarse de esta manera, Lahy le confiere a la psicofisiología el rol de árbitro social (Ribeill 1980), racional y neutral y, al mismo tiempo, legitima la subordinación del ingeniero de métodos a la ciencia. Además, la ciencia cumple la función de pacificar las relaciones sociales entre "patrones y trabajadores" (p.X).

\subsection{Síntesis}

Los límites de la psicotécnica se encuentran ampliamente informados hoy en día en la literatura (ver por ejemplo, Vatin, 1999). La evolución de los métodos psicotécnicos a partir de la segunda mitad de los años 30 ha contribuido a su más amplia difusión en las empresas (Le Bianic, 2007). Trabajando en la elaboración de una ciencia humana 
aplicada - de acuerdo con los principios sociales, políticos y económicos del momentoLahy no ha participado de las potenciales brechas entre los resultados del método de los tests y el análisis del trabajo que tanto le importaba. Los tests se han convertido en medios independientes y autónomos (Moutet, 2004).

Del mismo modo, Lahy no ha tomado el tiempo de considerar las capacidades de los obreros para transformar su trabajo y condiciones. La búsqueda del "punto de equilibrio" al que hace referencia en su crítica al método taylorista, sería para él la obra del científico, que sería el único capaz de proveer las bases para un compromiso organizacional renovado. En otros términos, se trata de sustituir al empirismo del método de Taylor, el rigor de un razonamiento científico llevado adelante por un tercero ilustrado, a su vez testigo en deuda con la sociedad y toda la humanidad.

\section{Actualidad acerca de las preguntas y elementos de discusión}

Las observaciones de Lahy han abierto el ejercicio de la psicología a perspectivas de aplicación prácticas, y permiten hacer nuevas preguntas relativas a las relaciones entre los diferentes protagonistas ligados a la intervención. A raíz de eso, resuena hoy en día junto a una demanda social renovada hacia la psicología del trabajo, concentrándose sobre las cuestiones de la calidad de vida en el trabajo, el "burn-out", e incluso el "boreout".

De hecho, la organización del trabajo contemporánea se enfrenta con desafíos ligados en particular a la revolución numérica. Ante estas condiciones en las que se renueva la uniformización del trabajo - a través de las buenas prácticas, el aumento de las normativas, el control de las prácticas- y de sus efectos sobre el trabajo y los profesionales mismos, emergen propuestas que tienden a retomar el proyecto profiláctico de Lahy. En concordancia con las demandas actuales de las empresas y la concepción de un sujeto cercano a una perspectiva cognitiva de las emociones, ciertas propuestas científicas se desprenden de un modo cada vez más claro. Las conductas positivas en el trabajo (por ejemplo, Bierhoff, 2002), la justicia organizacional (Steiner, 2012), el management benevolente (Colombat, 2016), el bienestar en el trabajo (Sovet, 2016), la intervención participativa (Ceccaldi, 2015), pasando por alto el análisis del trabajo, presentan soluciones científicas "positivas" ante problemas concretos y prácticos del trabajo cotidiano. La psicología del trabajo se encuentra entonces organizando las formas de ser, de actuar y de reaccionar ante situaciones individuales y colectivas, con el objeto de reconocer y considerar al otro para que "favorezca un funcionamiento armonioso de los grupos y las organizaciones" (Bernaud, Desrumaux \& Guédon, 2016, p. 2).

Estas perspectivas invierten la posición dominante compasiva del sufrimiento en el trabajo, hasta ahora muy presente. Pero en este movimiento pendular entre sufrimiento y bienestar, el tratamiento del trabajador por la ciencia positiva no modifica en nada la tendencia higienista (Clot, 2010). No son los profesionales - con su iniciativa para transformar la organización del trabajo, la tarea, los instrumentos, los recursos psicosociales - que son convocados para cuidar su trabajo, su oficio, su actividad, su salud. Los dispositivos de intervención se organizan frecuentemente a partir de cuestionarios y entrevistas, de acompañamiento individual y/o colectivo (ver 
por ejemplo, Lhotelier, Arnoux \& Bernaud, 2016). El trabajo, o más exactamente la representación que el sujeto se hace del mismo, "es solo un aspecto de la vida, en interacción con los otros" (p. 241). La centralidad del trabajo (Dejours \& Gernet, 2016), o su función psicológica (Clot, 2008) se repliegan, el análisis del trabajo, elemento esencial para Lahy, es ignorado, y las relaciones sociales, fundamentales en los ambientes de trabajo, se desprecian.

Es probablemente necesario encontrar una alternativa, y sobretodo, descubrirla junto a los primeros involucrados. Pero ¿qué es un "punto de equilibrio?, ¿Una relación armoniosa entre placer y sufrimiento, bienestar y maltrato, reconocimiento y negación, pasión por su trabajo e indiferencia? En nuestro caso, consideramos el equilibrio como "cooperación conflictiva" (Bonnefond, 2016 ; Clot, 2010 ; Trentin, 2012) centrada sobre la calidad del trabajo, los problemas concretos encontrados en el ejercicio cotidiano del trabajo. En este caso, el psicólogo del trabajo construye un dispositivo de co-análisis adaptado al pedido y regulado por las preocupaciones concretas de los profesionales que participan del análisis- permitiendo así alimentar a través de múltiples voces y múltiples niveles jerárquicos aquello que cuenta para los profesionales afín de llevar adelante un trabajo de calidad. El postulado - que es también el resultado de investigaciones previas (ver por ejemplo, Clot, 2008, 2010) - es el siguiente : el "hacer bien" ${ }^{4}$ subordina el "bien-estar". El precio de la renovación del compromiso social y organizacional probablemente sea ése, es decir sin "hacer trampa" con lo real. Sobre este punto es probable que Lahy, tan respetuoso del "terreno" y sus problemas, estaría de acuerdo con nosotros.

\section{BIBLIOGRAFÍA}

Bernaud, J.-L., Desrumeaux, P., \& Guédon, D. (Sous la dir.) (2016). Psychologie de la bientraitance professionnelle. Concepts, modèles et dispositifs. Paris : Dunod.

Bierhoff, H.-W. (2002). Prosocial Behavior. Hove: Psychology Press.

Bonnefond, J.-Y. (2016). L'intervention dans l'organisation en clinique de l'activité. Le dispositif «DQT» RENAULT à l'usine de Flins. Thèse de doctorat en psychologie. Cnam, Paris.

Ceccaldi, J. (2015). Pour un travail soigné dans une démarche participative. Paris : Lamarre.

Clot, Y. (2008). Travail et pouvoir d'agir. Paris : PUF.

Clot, Y. (2010). Le travail à cœur. Pour en finir avec les risques psychosociaux. Paris : La Découverte.

Cohen, Y. (2001). Organiser à l'aube du taylorisme. La pratique d'Ernest Mattern chez Peugeot, 1906-1919. Besançon : Presses Universitaires de Franche-Comté.

Colombat, P. (2016). Bientraitance, démarche participative et qualité de vie au travail : une application en milieu hospitalier. In J.-L Bernaud, P. Desrumaux \& D. Guédon (Sous la dir.), Psychologie de la bientraitance professionnelle. Concepts, modèles et dispositifs (pp. 71-83). Paris : Dunod. Dejours, C. \& Gernet, I. (2016). Psychopathologie du travail. Paris : Elsevier Masson. https://doi.org/ 10.3917/trav.036.0075 
Fridenson, P. (1987). Un tournant taylorien de la société française (1904-1918). Annales. Économies, Sociétés, Civilisations, 42(5), 1031-1060. https://doi.org/10.3406/ahess.1987.283435

Friedmann, G. (1946). Problèmes humains du machinisme industriel. Paris : Gallimard.

Gouarné, I. (2013). L'introduction du marxisme en France. Philosoviétisme et sciences humaines (1920-1939). Rennes : Presses universitaires de Rennes.

Hatzfeld (2011). De l'usure des corps au grippage de l'usine. L'histoire des ateliers automobiles éclairée par Simondon (XXè siècle). In L. Guignard, P. Raggi \& E. Thévenin (Sous la dir.), Corps et machines à l'âge industriel (pp. 137-149). Rennes : Presses Universitaires de Rennes.

Huteau, M. (2002). Psychologie, psychiatrie et société sous la troisième République. La biocratie d'Édouard Toulouse (1865-1947). Paris: L'Harmattan.

Lahy, J.-M. (1905). Les conditions psycho-physiologiques de l'aptitude au travail dactylographique. Journal de Physiologie et de Pathologie Générale, 15, 826-834.

Lahy, J.-M. (1909). Les conflits du travail. Hommes et femmes typographes. Revue Socialiste, 49, 289-312.

Lahy, J.-M. (1910). Recherches sur les conditions du travail des ouvriers typographes composant à la machine dite Linotype. Bulletin de l'inspection du travail et de l'hygiène industrielle, 1-2, 45-103.

Lahy, J.-M. (1913). La supériorité professionnelle chez les conducteurs de tramways dans ses rapports avec la consommation d'énergie. La technique moderne, 11, 388-390.

Lahy, J.-M. (1916). Le système Taylor et la physiologie du travail professionnel. Paris : Masson.

Le Bianic, T. (2004). Le Conservatoire des Arts et Métiers et la « machine humaine ». Naissance et développement des sciences de l'homme au travail au Cnam (1910-1990). Revue d'Histoire des Sciences Humaines, 2(11), 185-214. https://doi.org/10.3917/rhsh.011.0185

Le Bianic, T. (2007). Pratiques et identités professionnelles des psychologues du travail en France de l'entre-deux-guerres à nos jours. Une perspective socio-historique. Bulletin de Psychologie, 487(1), 71-81. https://doi.org/10.3917/bupsy.487.0071

Lhotellier, L., Arnoux, C., \& Bernaud, J.-L. (2016). Implanter un dispositif pour développer le sens du travail et de la vie. In J.-L Bernaud, P. Desrumaux \& D. Guédon (Sous la dir.), Psychologie de la bientraitance professionnelle. Concepts, modèles et dispositifs (pp. 229-243). Paris : Dunod.

Moutet, A. (2004). La psychologie appliquée à l'industrie : un moyen de définition de l'aptitude ou de l'inaptitude? (de 1930 à 1960). In C. Omnès \& A.S. Bruno (Sous la dir.), Les mains inutiles. Inaptitude au travail et emploi en France (p. 83-101). Paris : Editions Belin.

Paicheler, G. (1992). L'invention de la psychologie moderne. Paris : L'Harmattan.

Perrot, M. (1983). De la manufacture à l'usine en miettes. Le Mouvement Social, 125, 3-12. https:// doi.org/10.2307/3777679

Ribeill, G. (1980). Les débuts de l'ergonomie en France à la veille de la Première Guerre Mondiale. Le Mouvement Social, 113, 3-36. https://doi.org/10.2307/3778151

Steiner, D. D. (2012). Équité et justice au travail. In J. Allouche (Sous la dir.), Encyclopédie des ressources humaines (pp. 539- 545). Paris : Vuibert.

Sovet, L. (2016). Mesurer et modéliser le bien-être au travail. In J.-L Bernaud, P. Desrumaux \& D. Guédon (Sous la dir.), Psychologie de la bientraitance professionnelle. Concepts, modèles et dispositifs (pp. 55-65). Paris : Dunod. 
Trentin, B. (2012). La cité du travail : la gauche et la crise du fordisme. Paris : Fayard.

Turbiaux, M. (1999). Contribution à l'histoire des débats de la psychologie du travail en France. In Y. Clot (Sous la dir.), Les histoires de la psychologie du travail. Approche pluridisciplinaire (pp. 33-71). Paris : Octarès Editions.

Turbiaux, M. (2006). J.-M. Lahy (1872-1943) et l'orientation professionnelle. Bulletin de Psychologie, 482(2), 217-235. https://doi.org/10.3917/bupsy.482.0217

Vatin, F. (1999). De la naissance de la psychologie appliquée au débat sur le taylorisme, autopsie d'un échec : le cas français (1890-1920). In Y. Clot (Sous la dir.), Les histoires de la psychologie du travail. Approche pluridisciplinaire (pp. 91-111). Paris : Octarès Editions.

Vatin, F. (2008). Les « sciences du travail » : une tentative de résolution positiviste de la question sociale (1890-1914). Bulletin de Psychologie, 61(4), 331-340. https://doi.org/10.3917/bupsy.496.0331

\section{NOTAS}

1. Ecole Pratique des Hautes Etudes - EPHE.

2. La EPHE recibe auditores sin condiciones previas de diploma alguno.

3. Cabe señalar que este libro fue escrito antes de la Primera Guerra Mundial. La crítica realizada por Lahy, luego de la difusión del modelo taylorista al finalizar la Primera Gue- rra Mundial, resulta aún más justificada.

4. N.T.: La expresión francesa "bien-être" puede traducirse como "bienestar" o como "estar bien".

\section{AUTORES}

\section{JEAN-LUC TOMÁS}

CRTD EA4132, Cnam, Paris, Équipe Psychologie du travail et clinique de l'activité 41, rue Gay Lussac 75005 Paris France jean-luc.tomas@lecnam.net

\section{MARIA IANEVA}

CRTD EA4132, Cnam, Paris, Équipe Psychologie du travail et clinique de l'activité 41, rue Gay Lussac 75005 Paris France maria.ianeva@lecnam.net 